\title{
Analysis of clinical features and pulmonary CT features of coronavirus disease 2019 (COVID-19) patients with diabetes mellitus
}

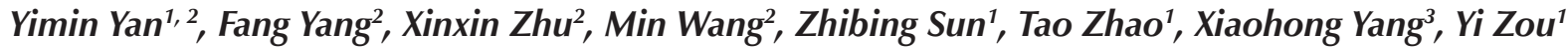 \\ ${ }^{1}$ Department of Endocrinology, Xiaogan Hospital Affiliated to Wuhan University of Science and Technology, \\ The Central Hospital of Xiaogan, Xiaogan, Hubei, China \\ ${ }^{2}$ Medical College of Wuhan University of Science and Technology, Wuhan, China \\ ${ }^{3}$ Department of Gynaecological Endocrinology, Xiaogan Hospital Affiliated to Wuhan University of Science and Technology, \\ The Central Hospital of Xiaogan, Xiaogan, Hubei, China
}

\begin{abstract}
Introduction: The objective of this paper was to investigate the clinical features and pulmonary CT imaging features of COVID-19 patients with diabetes mellitus.

Material and methods: From January 16, 2020 to March 28, 2020, among the 568 cases of COVID-19 patients diagnosed in Xiaogan Central Hospital, 64 cases of COVID-19 patients with diabetes were selected as the diabetic group, and 64 cases of COVID-19 patients with age and gender matching without diabetes were selected as the non-diabetic group, and their clinical data and pulmonary CT characteristics were retrospectively analysed.

Results: Compared with the non-diabetic group, the proportion of patients in the diabetic group with chronic underlying disease was higher, and they were in more a serious condition at admission. Inflammation index and characteristics of glycolipid metabolism results showed that COVID-19 patients with diabetes mellitus were more likely to have elevated inflammatory markers and hypercoagulability, accompanied by hypoproteinaemia and glucose and lipid metabolism disorders. Treatment and clinic outcome results showed that the time of nucleic acid turning negative in the diabetic group was significantly longer than that in the non-diabetic group. Radiological data showed that COVID-19 combined with diabetes prolonged the time of detoxification in patients.

Conclusion: COVID-19 patients with diabetes mellitus and chronic hypertension are associated with increased inflammatory markers and disorders of glucose and lipid metabolism. These patients tend to develop serious diseases, especially the rapid progression of CT lesions in the lungs of patients with a wide range of involvement, and prolonged absorption and detoxification time. (Endokrynol Pol 2020; 71 (5): 367-375)
\end{abstract}

Key words: COVID-19; diabetes; CT image; detoxification time

\section{Introduction}

Coronaviruses are enveloped RNA viruses that are widely distributed in humans, other mammals, and birds, and which cause respiratory, intestinal, liver, and neurological diseases [1-2]. At present, six kinds of coronaviruses are known to cause human diseases [3]. In view of the high prevalence and wide distribution of coronaviruses, the huge genetic diversity, and frequent recombination of their genomes, as well as the increasing human-animal interface activities that induce frequent cross-species infections and occasional spillovers, new coronaviruses may appear periodically in human beings [4-5]. In December 2019, the outbreak of severe acute respiratory syndrome coronavirus 2 (SARS-CoV-2) was first reported in Wuhan, China, which had a huge impact on China and the world. The disease caused by SARS-CoV-2 was named as coronavirus disease 2019 (COVID-19) [6]. In clinical practice, we have observed many severe or critically COVID-19 patients with typical clinical manifestations of shock, including cold extremities and weak peripheral pulse. Even in the absence of obvious hypotension, many patients present with severe metabolic acidosis, suggesting the possibility of microcirculatory dysfunction [7]. Furthermore, in addition to severe lung injury, some patients also have impaired liver and kidney functions [8], which suggests that COVID-19 may be associated with chronic underlying diseases.

As a representative of chronic basic diseases, diabetes is a disease involving multiple metabolic disorders, characterised by high blood glucose concentration and 
inhibition of glucose oxidation, resulting in increased lipid metabolism, which in turn causes in hyperlipidaemia, hyperinsulinaemia, hypercoagulable symptoms, and other clinical symptoms [9]. At the same time, studies have shown that $71.4 \%$ of non-survivors of COVID-19 met the significant disseminated intravascular coagulation grade $(\geq 5$, meeting the standards of the International Society on Thrombosis and Haemostasis) and showed a significant correlation between abnormal coagulation results and poor prognosis at the later stage of COVID-19 disease [10]. An association between COVID-19 and diabetes has been suggested, but there is currently very limited research on the association between COVID-19 and diabetes. Therefore, the focus of this present study was to explore the correlation between COVID-19 and diabetes mellitus in terms of clinical features and pulmonary CT features. In addition, the clinical indexes, inflammatory indexes, glucose and lipid metabolism, and detoxification time of patients with COVID-19 combined with diabetes were studied. This article aims to make up for the limitations of the study of COVID-19 patients with diabetes, and at the same time provide a certain theoretical basis and clinical guidance for the treatment of COVID-19 patients with diabetes.

\section{Material and methods}

\section{General data collection}

The patients' epidemiological data, medical history, contact history, symptoms and signs, laboratory examination, complications, clinical outcomes, CT imaging data, and treatment plan were extracted from electronic medical records. The date of onset of the disease was the date of the first symptom. The team analysed all the data, and it was double checked by two doctors. Nucleic acid detection was carried out by Xiaogan Central Hospital. At the time of admission, throat swab specimens were obtained from the patient's upper respiratory tract and stored in a virus transport medium. Total RNA was extracted within two hours using the RNA separation kit of respiratory tract samples. Suspected cases were selected according to the diagnostic criteria for COVID-19 pneumonia [11]:

1. Epidemiological history:

- travel or residence history in Wuhan or other areas with continuous transmission of local cases within 14 days before the onset of the COVID-19 disease;

- contact with patients with fever or respiratory symptoms from Wuhan or other areas where local cases continued to spread within 14 days before the onset of the COVID-19 disease;

- clusters of COVID-19 disease or epidemiological association with COVID-19 infection.

2. Clinical manifestations:

- fever;

- with the above-mentioned imaging characteristics of pneumonia;

- normal or decreased total number of white blood cells in the early stage of COVID-19 disease, or reduced lymphocyte count. Suspected cases could be diagnosed if any one of them had an epidemiological history and conformed to any two of the clinical manifestations.

Inclusion criteria for patients: 1 - suspected cases of COVID-19 pneumonia; 2 - COVID-19 nucleic acid RT-PCR detection of sputum, throat swabs, lower respiratory tract secretions, and other specimens were positive; 3 - diabetes was diagnosed according to the 1999 World Health Organisation (WHO) diagnostic criteria for diabetes [12]. This study was approved by the ethics committee of Xiaogan Central Hospital (ethics no.: XGLY2020-03-29) and conformed to the declaration of Helsinki.

\section{Detection methods}

After admission, fasting venous blood was collected in the morning and sent to the laboratory for blood routine examination and biochemical examination; the operation was carried out according to our laboratory instructions.

\section{CT image collection}

Two experienced physicians were employed to review the films, and they conducted quantitative accounting according to the distribution, location, size, morphology, edge, density, and pulmonary manifestations of the lesions.

\section{Statistical analysis}

Classification variables were expressed as frequency and percentage, continuous variables were expressed as average, and quantitative data of non-normal distribution were calculated by quartile. Chi-square test and Fisher exact test were used in the two groups of data, and t test or Mann-Whitney U test were used to analyse continuous variables. SPSS21.0 software was used for all statistical analysis. $P<0.05$ was considered statistically significant.

\section{Results}

From January 16, 2020 to March 28, 2020, among the 568 cases of COVID-19 (data not shown) patients diagnosed in Xiaogan Central Hospital, 64 cases of COVID-19 patients with diabetes were selected as the diabetic group (hereinafter referred to as C-DM), and 64 cases of COVID-19 patients with age and gender matching without diabetes were selected as the non-diabetic group (hereinafter referred to as Non-C-DM) to study the clinical characteristics of patients with COVID-19 combined with diabetes mellitus - 128 cases in total. Clinical characteristics of C-DM and Non-C-DM cases were summarised in Table 1 . In detail, among the 128 COVID-19 patients, the median age was 58 years [IQR (50-70)]; there were 74 males $(57.81 \%)$ and 54 females $(42.19 \%)$. The most common symptoms were fever $(85.16 \%)$ and cough $(71.09 \%)$; followed by dyspnoea $(34.38 \%)$, fatigue (31.25\%), and expectoration (25.78\%); meanwhile diarrhoea $(7.03 \%)$, myalgia $(5.47 \%)$, dizziness $(2.34 \%)$, sore throat $(2.34 \%)$, nausea $(1.56 \%)$, and conjunctival congestion $(0.78 \%)$ were relatively rare. The vast majority of patients had a clear contact history, including Wuhan tourism history (23.44\%), Wuhan residential history $(10.16 \%)$, and contact history with confirmed patients $(21.88 \%)$; the proportion of patients with non-clear contact history was $44.53 \%$. Hypertension $(39.06 \%$ ) was the most common chronic disease, followed by cardiovascular disease $(8.59 \%)$ and cerebrovascular disease $(2.34 \%)$. 
Table 1. Clinical characteristics of patients with diabetes (C-DM) and without diabetes (Non-C-DM)

\begin{tabular}{|c|c|c|c|c|}
\hline Characteristics & $\begin{array}{l}\text { All patients } \\
(\mathrm{n}=568)\end{array}$ & $\begin{array}{c}\text { Non-C-DM } \\
(\mathrm{n}=504)\end{array}$ & $\begin{array}{c}\text { C-DM } \\
(n=64)\end{array}$ & p value \\
\hline $\begin{array}{l}\text { Age (years) } \\
\text { Median (IOR) }\end{array}$ & $52(43-63)$ & $52(42-62)$ & $58(50-70)$ & 0.004 \\
\hline \multicolumn{5}{|l|}{$\begin{array}{l}\text { Age groups (years) } \\
\text { n (\%) }\end{array}$} \\
\hline$\leq 39$ & $107(18.84)$ & $101(20.04)$ & $6(9.38)$ & 0.040 \\
\hline $40-49$ & $119(20.95)$ & $111(22.02)$ & $8(12.5)$ & 0.078 \\
\hline $50-59$ & $158(27.82)$ & $137(0.27)$ & $21(32.81)$ & 0.344 \\
\hline $60-69$ & $80(14.08)$ & $67(13.29)$ & $13(20.31)$ & 0.128 \\
\hline$\geq 70$ & $104(18.31)$ & $88(17.46)$ & $16(25.00)$ & 0.142 \\
\hline \multicolumn{5}{|l|}{$\begin{array}{l}\text { Gender } \\
\text { n (\%) }\end{array}$} \\
\hline Male & $309(54.40)$ & $272(53.97)$ & $37(57.81)$ & 0.561 \\
\hline Female & $259(45.60)$ & $232(46.03)$ & $27(42.19)$ & 0.561 \\
\hline \multicolumn{5}{|l|}{ BMI $\left[\mathrm{kg} / \mathrm{m}^{2}\right]$} \\
\hline$\leq 18.5$ & $21(3.70)$ & $19(3.77)$ & $2(3.13)$ & 1.000 \\
\hline $18.5<\mathrm{BMI} \leq 24$ & $288(50.70)$ & $265(52.58)$ & $23(35.94)$ & 0.012 \\
\hline $24<\mathrm{BMI} \leq 28$ & $193(33.98)$ & $167(33.13)$ & $26(40.63)$ & 0.233 \\
\hline $28<\mathrm{BMI} \leq 32$ & $52(9.15)$ & $42(8.33)$ & $10(15.63)$ & 0.057 \\
\hline$>32$ & $8(1.41)$ & $7(1.39)$ & $1(1.56)$ & 1.000 \\
\hline \multicolumn{5}{|l|}{$\begin{array}{l}\text { Exposure history } \\
\text { n (\%) }\end{array}$} \\
\hline History of residence in Wuhan & $60(10.56)$ & $56(11.11)$ & $4(6.25)$ & 0.329 \\
\hline Wuhan tourism history & $126(22.18)$ & $115(22.82)$ & $11(17.19)$ & 0.307 \\
\hline Contact history with confirmed patients & $139(24.47)$ & $122(24.21)$ & $17(26.56)$ & 0.680 \\
\hline Denied a clear contact history & $243(42.78)$ & $211(41.87)$ & $32(50.00)$ & 0.215 \\
\hline \multicolumn{5}{|l|}{$\begin{array}{l}\text { Comorbidities } \\
\text { n (\%) }\end{array}$} \\
\hline Smoking & $34(5.99)$ & $28(5.56)$ & $6(9.38)$ & 0.225 \\
\hline Hypertension & $139(24.47)$ & $107(21.23)$ & $32(50.00)$ & 0.000 \\
\hline Cardiovascular disease & $31(5.46)$ & $23(4.56)$ & $8(12.50)$ & 0.008 \\
\hline Cerebrovascular disease & $14(2.46)$ & $11(2.18)$ & $3(4.69)$ & 0.430 \\
\hline Chronic pulmonary disease & $23(4.05)$ & $20(3.97)$ & $3(4.69)$ & 1.000 \\
\hline Chronic kidney diseases & $4(0.70)$ & $3(0.60)$ & $1(1.56)$ & 0.938 \\
\hline Chronic liver disease & $22(3.87)$ & $22(4.37)$ & $0(0)$ & $\mathrm{N} / \mathrm{A}$ \\
\hline Rheumatic immune diseases & $6(1.06)$ & $6(1.19)$ & $0(0)$ & $\mathrm{N} / \mathrm{A}$ \\
\hline Malignancies & $17(2.99)$ & $12(2.38)$ & $5(7.81)$ & 0.016 \\
\hline \multicolumn{5}{|l|}{$\begin{array}{l}\text { Clinical symptoms } \\
\text { n (\%) }\end{array}$} \\
\hline Fever & $507(89.26)$ & $453(89.88)$ & $54(84.38)$ & 0.180 \\
\hline Cough & $392(69.01)$ & $339(67.26)$ & $53(82.81)$ & 0.011 \\
\hline Expectoration & $144(25.35)$ & $124(24.60)$ & $20(31.25)$ & 0.250 \\
\hline Dyspnoea & $202(35.56)$ & 175 (34.72) & $27(42.19)$ & 0.240 \\
\hline Conjunctival congestion & $1(0.18)$ & $0(0)$ & $1(1.56)$ & $\mathrm{N} / \mathrm{A}$ \\
\hline Pharyngalgia & $30(5.28)$ & $27(5.36)$ & $3(4.69)$ & 1.000 \\
\hline Dizziness & $8(1.41)$ & $7(1.39)$ & $1(1.56)$ & 1.000 \\
\hline Myalgia & $28(4.93)$ & $25(4.96)$ & $3(4.69)$ & 1.000 \\
\hline
\end{tabular}


Table 1. Clinical characteristics of patients with diabetes (C-DM) and without diabetes (Non-C-DM)

\begin{tabular}{|c|c|c|c|c|}
\hline Characteristics & $\begin{array}{l}\text { All patients } \\
(\mathrm{n}=568)\end{array}$ & $\begin{array}{c}\text { Non-C-DM } \\
(\mathrm{n}=504)\end{array}$ & $\begin{array}{c}\text { C-DM } \\
(\mathrm{n}=64)\end{array}$ & p value \\
\hline Fatigue & $185(32.57)$ & $164(32.54)$ & $21(32.81)$ & 0.965 \\
\hline Nausea or vomiting & $14(2.46)$ & $12(2.38)$ & $2(3.13)$ & 1.000 \\
\hline Diarrhoea & $28(4.93)$ & $24(4.76)$ & $4(6.25)$ & 0.832 \\
\hline Temperature $\geq 37.3^{\circ} \mathrm{C}$ & $147(25.88)$ & $130(25.79)$ & $17(26.56)$ & 0.895 \\
\hline $\mathrm{SpO}_{2} \leq 93 \%$ & $90(15.85)$ & $69(13.69)$ & $21(32.81)$ & 0.000 \\
\hline$H R>100$ & $89(15.67)$ & $73(14.48)$ & $16(25.0)$ & 0.029 \\
\hline
\end{tabular}

$\mathrm{IQR}$ — interquartile range; $\mathrm{BMI}$ — body mass index; $\mathrm{SpO}_{2}$ — oxygen saturation; HR — heart rate

As shown in Table 1, compared with the Non-C-DM group, the number of patients with chronic underlying diseases complicated with hypertension in the C-DM group increased significantly (50.00\% vs. $28.13 \%$, $\mathrm{p}<0.05)$. Cough symptoms were more common in the C-DM group $(82.81 \%$ vs. $59.38 \%, \mathrm{p}<0.05)$. In addition, patients in the C-DM group were more likely to have decreased oxygen saturation and tachyarrhythmia at admission, in which oxygen saturation $\leq 93 \%(32.81 \%$ vs. $9.38 \%, \mathrm{p}<0.05)$ and heart rate $>100 \mathrm{bpm}(25.00 \%$ vs. $10.94 \%, \mathrm{p}<0.05)$, indicating that patients in the C-DM group were in more a serious condition at admission.

Inflammation index and characteristics of glycolipid metabolism of C-DM and Non-C-DM cases are summarised in Table 2. In particular, in comparison

Table 2. Laboratory test of patients with diabetes (C-DM) and without diabetes (Non-C-DM)

\begin{tabular}{|c|c|c|c|c|c|}
\hline & $\begin{array}{l}\text { Normal } \\
\text { range }\end{array}$ & $\begin{array}{l}\text { All patients } \\
(\mathrm{n}=128)\end{array}$ & $\begin{array}{c}\text { Non-C-DM } \\
(n=64)\end{array}$ & $\begin{array}{c}\text { C-DM } \\
(n=64)\end{array}$ & p value \\
\hline \multicolumn{6}{|l|}{ Blood routine } \\
\hline White blood cell count $\left[\times 10^{9} / \mathrm{L}\right]$ & $3.5-9.5$ & $5.07(3.76-6.74)$ & $4.64(3.40-5.97)$ & $5.65(4.44-7.37)$ & 0.006 \\
\hline Red blood cell count $\left[\times 10^{12} / \mathrm{L}\right]$ & $3.92-5.61$ & $4.39(3.97-4.76)$ & $4.33(3.83-4.71)$ & 4.47 (3.99-4.77) & 0.563 \\
\hline Haemoglobin & $115-150$ & $137(118-148)$ & $138.00(118.00-152.00)$ & $135.50(117.75-146.00)$ & 0.335 \\
\hline Neutrophil $\left[\times 10^{9} / L\right]$ & $1.5-6.3$ & $3.45(2.43-4.94)$ & $3.08(2.03-4.10)$ & $4.15(2.75-5.86)$ & 0.003 \\
\hline Lymphocyte $\left[\times 10^{9} / \mathrm{L}\right]$ & $1.1-3.2$ & $0.95(0.70-1.38)$ & $0.98(0.73-1.42)$ & $0.93(0.64-1.32)$ & 0.255 \\
\hline Platelets $\left[\times 10^{9} / \mathrm{L}\right]$ & $125-350$ & $166(124-222)$ & $153.00(120.00-207.00)$ & $187(130.75-267.75)$ & 0.053 \\
\hline \multicolumn{6}{|l|}{ Blood coagulation } \\
\hline Active partial thrombin time (APTT) & $23-45$ & $31.00(28.20-33.70)$ & $31.85(29.25-34.10)$ & $30.50(27.75-33.45)$ & 0.140 \\
\hline PT & $9-14$ & $12.70(11.90-13.70)$ & $13.00(12.23-13.78)$ & 12.60 (11.75-13.65) & 0.259 \\
\hline D-dimer (SDD) & $0-1$ & $0.30(0.25-0.48)$ & $0.28(0.23-0.36)$ & $0.34(0.26-0.62)$ & 0.007 \\
\hline TP & $65-85$ & $67.20(63.70-72.63)$ & $66.90(63.70-72.45)$ & $67.75(63.73-72.73)$ & 0.864 \\
\hline ALB & $40-55$ & $38.00(35.18-40.73)$ & $38.95(37.13-41.15)$ & $36.80(34.83-39.63)$ & 0.007 \\
\hline ALT & $7-40$ & $20.00(13.00-33.25)$ & $19.00(12.75-34.25)$ & $21.00(14.00-32.25)$ & 0.639 \\
\hline AST & $13-35$ & $24.00(18.75-34.25)$ & $24.50(19.75-32.25)$ & $24.00(16.75-41.00)$ & 0.888 \\
\hline Total bilirubin & $0-23$ & $12.05(9.48-16.35)$ & $12.50(9.50-15.98)$ & $11.95(9.18-16.38)$ & 0.884 \\
\hline Urea nitrogen & $2.6-7.5$ & $4.55(3.30-5.83)$ & $4.60(3.50-5.60)$ & $4.40(3.10-6.45)$ & 0.691 \\
\hline Creatinine & $41-73$ & $70.35(60.15-85.25)$ & $71.80(62.98-86.78)$ & $67.40(56.03-84.18)$ & 0.168 \\
\hline eGFR & & 94.74 (81.58-109.55) & 93.55 (79.69-105.75) & $102.58(84.49-115.63)$ & 0.076 \\
\hline $\mathrm{LDH}$ & $120-250$ & $245.50(196.75-324.25)$ & $226.50(194.75-280.25)$ & $271.50(210.50-347.0)$ & 0.019 \\
\hline ALP & $40-150$ & $69.00(59.00-85.00)$ & $69.00(60.00-84.25)$ & $67.00(57.75-87.25)$ & 0.924 \\
\hline FPG & $3.89-6.11$ & $6.19(5.50-9.26)$ & $5.58(5.19-6.00)$ & $8.85(6.51-11.16)$ & 0.000 \\
\hline $\mathrm{TC}$ & $2.9-5.17$ & $3.58(2.93-4,18)$ & $3.53(2.93-4.13)$ & $3.62(2.95-4.25)$ & 0.708 \\
\hline TG & $0.23-1.7$ & $1.33(1.00-1.86)$ & $1.11(0.94-1.60)$ & $1.54(1.12-2.29)$ & 0.000 \\
\hline
\end{tabular}


Table 2. Laboratory test of patients with diabetes (C-DM) and without diabetes (Non-C-DM)

\begin{tabular}{|c|c|c|c|c|c|}
\hline & $\begin{array}{c}\text { Normal } \\
\text { range }\end{array}$ & $\begin{array}{l}\text { All patients } \\
(\mathrm{n}=128)\end{array}$ & $\begin{array}{c}\text { Non-C-DM } \\
(\mathrm{n}=64)\end{array}$ & $\begin{array}{c}\text { C-DM } \\
(\mathrm{n}=64)\end{array}$ & p value \\
\hline \multicolumn{6}{|c|}{ Four items of chest pain } \\
\hline CKMB & $0-4$ & $2.48(1.70-3.70)$ & $2.20(1.74-3.11)$ & $2.60(1.66-3.85)$ & 0.414 \\
\hline BNP & $0-125$ & 227.00 (93.50-740.75) & $247.00(158.50-652.50)$ & $191.50(54.75-820.50)$ & 0.296 \\
\hline cTnl & $0.04-0.5$ & $0.06(0.03-0.08)$ & $0.06(0.04-0.09)$ & $0.06(0.03-0.08)$ & 0.670 \\
\hline \multicolumn{6}{|c|}{ Infection-related indicators } \\
\hline РCT & $0-0.5$ & $0.17(0.12-0.29)$ & $0.14(0.11-0.28)$ & $0.19(0.14-0.29)$ & 0.096 \\
\hline CRP & $0-3$ & $19.61(4.61-45.40)$ & $15.90(3.27-35.19)$ & $24.43(6.47-68.05)$ & 0.029 \\
\hline ESR & $0-20$ & $39.50(25.00-74.75)$ & $29.00(21.50-60.00)$ & $55.00(38.50-80.00)$ & 0.045 \\
\hline \multicolumn{6}{|c|}{ Blood gas analysis } \\
\hline $\mathrm{pH}$ & $7.35-7.45$ & $7.43(7.40-7.47)$ & $7.43(7.42-7.47)$ & $7.44(7.40-7.46)$ & 0.557 \\
\hline $\mathrm{PO}_{2}$ & $83-108$ & 80.15 (67.15-99.75) & $84.40(68.18-105.00)$ & 74.75 (66.10-90.45) & 0.793 \\
\hline $\mathrm{PCO}_{2}$ & $35-45$ & $38.05(32.55-41.73)$ & $39.25(36.03-42.45)$ & $36.30(30.80-40.75)$ & 0.166 \\
\hline LAC & $0.5-1.6$ & $1.55(1.10-2.00)$ & $1.20(0.09-1.68)$ & $1.80(1.30-2.90)$ & 0.112 \\
\hline
\end{tabular}

APTT — active partial thrombin time; PT — prothrombin time; TP — total protein; ALB — albumin propagated; ALT — alanine aminotransferase; AST — glutamates transaminase; eGFR — estimated glomerular filtration rate; LDH — lactate dehydrogenase; ALP — alkaline phosphatase; FPG — fasting plasma glucose;

$\mathrm{TC}$ — total cholesterol; TG — triglyceride; CKMB — creatinase myocardial band; BNP — N-terminal brain natriuretic peptide precursor; cTnl — cardiac troponin;

$\mathrm{PCT}$ - procalcitonin; CRP — C-reactive protein; ESR — Erythrocyte sedimentation rate; $\mathrm{PO}_{2}$ - oxygen partial pressure; $\mathrm{PCO}_{2}$ - partial pressure of carbon dioxide LAC — lactic acid

to the Non-C-DM group, C-DM group white blood cells [5.65 IQR (4.44-7.37) vs. $4.64(3.40-5.97)]$, PCT [0.19 IQR (0.14-0.29) vs. $0.14(0.11-0.28)]$, CRP (24.43 IQR (6.47-68.05) vs. 15.90 (3.27-35.19)], ESR [55.00 IQR (38.50-80.00) vs. $29.00(21.50-60.00)]$, SDD [0.34 IQR (0.26-0.62) vs. $0.28(0.23-0.36)]$, FPG [8.85 IQR (6.51-11.16) vs. 5.58 (5.19-6.00)], and TG [3.62 IQR (2.95-4.25) vs. 3.53 (2.93-4.13)] increased, and albumin [36.80 IQR (34.83-39.63) vs. 38.95 (37.13-41.15)] decreased. These results suggested that COVID-19 patients with diabetes mellitus are more likely to have elevated inflammatory markers and hypercoagulability, accompanied by hypoproteinaemia and glucose and lipid metabolism disorders.

Treatment and clinic outcome of C-DM and Non-C-DM cases are summarised in Table 3. In detail, compared with the Non-C-DM group, the proportion of C-DM-group patients with respiratory failure ( $37.50 \%$ vs. $7.81 \%$ ), requiring oxygen therapy $(48.44 \%$ vs. $31.25 \%)$, and a non-invasive ventilator $(17.19 \%$ vs. $3.13 \%$ ) were significantly higher. Meanwhile, the C-DM group critical illness rate $(28.13 \%$ vs. $3.13 \%)$ and the mortality rate $(15.63 \%$ vs. $3.13 \%)$ were also significantly higher than those in the Non-C-DM group. The median length of stay for all patients was 20 days, IQR (14-25). The length of stay in the C-DM group [22 IQR (16-27) vs. 17 (13-23)] was also relatively long, and the time of nucleic acid turning negative in the $\mathrm{C}$-DM group was significantly greater than that in Non-C-DM group [18 IQR (15-22) vs. 23 (18-30)].
Radiological data of C-DM and Non-C-DM cases are summarised in Table 4. Specifically, according to the distribution characteristics and the range of involvement of COVID-19 lung CT, we counted the imaging characteristics of lung CT in the first week of admission. Among all the patients, the proportion of lesions involving both lungs was $79.69 \%$, the proportion of lesion distribution simultaneously involving peripheral and central areas was $50.78 \%$, the proportion of lesion size of $>3 \mathrm{~cm}$ was $78.91 \%$, the proportion of three or more lesions was $77.34 \%$, and the proportion of lesion edge blur was $83.59 \%$. In terms of lung CT lesion involvement location, compared with the Non-C-DM group ( $54.69 \%$ vs. $28.13 \%$ ), the proportion of peripheral and central involvement was higher the C-DM group (62.50\% vs. 39.06\%). In terms of lung CT lesion morphology, the Non-C-DM group was mostly affected by plaque $(57.81 \%$ vs. $25.00 \%)$, while the lung lobes of C-DM group were often affected (32.81\% vs. $12.50 \%$ ). More importantly, we calculated the proportion of pulmonary CT progression at the first week and found that the C-DM group had significantly higher progession than the Non-C-DM group $(92.19 \%$ vs. $60.94 \%, \mathrm{p}<0.001)$, while the time of pulmonary $\mathrm{CT}$ absorption was significantly greater in the C-DM group than in the non-diabetic group [26 IQR (16-34) vs. 19 (15-23)], $\mathrm{p}<0.05$ (Fig. 1). This proved our hypothesis that COVID-19 combined with diabetes might prolong the time of detoxification in patients. 
Table 3. Treatment and clinical outcome of patients with diabetes (C-DM) and without diabetes (Non-C-DM)

\begin{tabular}{|c|c|c|c|c|}
\hline & $\begin{array}{l}\text { Number of cases } \\
\qquad(\mathrm{n}=128)\end{array}$ & $\begin{array}{c}\text { Non-C-DM } \\
(\mathrm{n}=64)\end{array}$ & $\begin{array}{c}\text { C-DM } \\
(\mathrm{n}=64)\end{array}$ & $\mathrm{p}$ value \\
\hline Oxygen n (\%) & $51(39.84)$ & $20(31.25)$ & $31(48.44)$ & 0.047 \\
\hline Non-invasive ventilator $\mathrm{n}(\%)$ & $13(10.16)$ & $2(3.13)$ & $11(17.19)$ & 0.019 \\
\hline Invasive ventilator n (\%) & $7(5.47)$ & $1(1.56)$ & $6(9.38)$ & 0.120 \\
\hline \multicolumn{5}{|l|}{$\begin{array}{l}\text { Complications } \\
\text { n (\%) }\end{array}$} \\
\hline Respiratory failure & $29(22.66)$ & $5(7.81)$ & $24(37.50)$ & 0.000 \\
\hline Heart failure & $5(3.91)$ & $2(3.13)$ & $3(4.69)$ & 1.000 \\
\hline Septic shock & $3(2.34)$ & $1(1.56)$ & $2(3.13)$ & 1.000 \\
\hline \multicolumn{5}{|l|}{$\begin{array}{l}\text { Clinical classification } \\
\text { n (\%) }\end{array}$} \\
\hline Ordinary & $80(62.50)$ & $52(81.25)$ & $28(43.75)$ & 0.000 \\
\hline Heavy & $28(21.88)$ & $10(15.63)$ & $18(28.13)$ & 0.087 \\
\hline Critical type & $20(15.63)$ & $2(3.13)$ & $18(28.13)$ & 0.000 \\
\hline \multicolumn{5}{|l|}{$\begin{array}{l}\text { Clinical outcome } \\
\text { n (\%) }\end{array}$} \\
\hline Discharge & $116(90.63)$ & $62(96.88)$ & $54(84.38)$ & 0.015 \\
\hline Death & $12(9.38)$ & $2(3.13)$ & $10(15.63)$ & 0.034 \\
\hline Length of stay & $20(14-25)$ & $17(13-23)$ & $22(16-27)$ & 0.029 \\
\hline
\end{tabular}

Table 4. Radiological data of patients with diabetes (C-DM) and without diabetes (Non-C-DM)

\begin{tabular}{|c|c|c|c|c|c|}
\hline & & $\begin{array}{l}\text { Number of cases } \\
\qquad(n=128)\end{array}$ & $\begin{array}{l}\text { Non-C-DM } \\
(\mathrm{n}=64)\end{array}$ & $\begin{array}{c}\text { C-DM } \\
(n=64)\end{array}$ & p value \\
\hline \multirow{3}{*}{ Lesion distribution } & Left lung & $8(6.25)$ & $7(10.94)$ & $1(1.56)$ & 0.068 \\
\hline & Right lung & $8(6.25)$ & $4(6.25)$ & $4(6.25)$ & 1.000 \\
\hline & Double lung & $102(79.69)$ & $49(76.56)$ & $53(82.81)$ & 0.380 \\
\hline \multirow{2}{*}{ Lesion location } & Periphery & $53(41.41)$ & $35(54.69)$ & $18(28.13)$ & 0.002 \\
\hline & Periphery and centre & $65(50.78)$ & $25(39.06)$ & $40(62.50)$ & 0.008 \\
\hline \multirow{3}{*}{ Lesion size [cm] } & $<1$ & $7(5.47)$ & $6(9.38)$ & $1(1.56)$ & 0.120 \\
\hline & $1 \sim 3$ & $10(7.81)$ & $7(10.94)$ & $3(4.69)$ & 0.323 \\
\hline & $>3$ & $101(78.91)$ & $47(73.44)$ & $54(81.38)$ & 0.129 \\
\hline \multirow{3}{*}{ Lesion form } & Patch & $53(41.41)$ & $37(57.81)$ & $16(25.00)$ & 0.000 \\
\hline & Lung segment & $36(28.13)$ & $15(23.44)$ & $21(32.81)$ & 0.238 \\
\hline & Lobe & $29(22.66)$ & $8(12.50)$ & $21(32.81)$ & 0.006 \\
\hline \multirow{3}{*}{ Number of lesions } & 1 & $13(10.16)$ & $8(12.50)$ & $5(7.81)$ & 0.380 \\
\hline & 2 & $6(4.69)$ & $4(6.25)$ & $2(3.13)$ & 0.676 \\
\hline & 3 or more & 99 (77.34) & $48(75.00)$ & 51 (79.69) & 0.526 \\
\hline \multirow{2}{*}{ Lesion margin } & Clear & $10(7.81)$ & $6(9.38)$ & $4(6.25)$ & 0.742 \\
\hline & Vague & $107(83.59)$ & $53(82.81)$ & 54 (81.38) & 0.811 \\
\hline \multirow{3}{*}{ Lesion density } & Ground glass & $48(37.50)$ & $24(37.50)$ & $24(37.50)$ & 1.000 \\
\hline & Substantiality & $3(2.34)$ & $2(3.13)$ & $1(1.56)$ & 1.000 \\
\hline & Mixed type & $68(53.13)$ & 35 (54.69) & $33(51.56)$ & 0.723 \\
\hline \multirow{3}{*}{$\begin{array}{l}\text { Extrapulmonary } \\
\text { manifestations }\end{array}$} & $\begin{array}{l}\text { Mediastinal } \\
\text { lymphadenopathy }\end{array}$ & 0 & 0 & 0 & $\mathrm{~N} / \mathrm{A}$ \\
\hline & Pneumothorax & 0 & 0 & 0 & N/A \\
\hline & Pleural effusion & $8(6.25)$ & $4(6.25)$ & $4(6.25)$ & 1.000 \\
\hline
\end{tabular}


Table 4. Radiological data of patients with diabetes (C-DM) and without diabetes (Non-C-DM)

\begin{tabular}{lcccc}
\hline & $\begin{array}{c}\text { Number of cases } \\
(\mathbf{n}=\mathbf{1 2 8})\end{array}$ & $\begin{array}{c}\text { Non-C-DM } \\
(\mathbf{n}=\mathbf{6 4})\end{array}$ & $\begin{array}{c}\text { C-DM } \\
(\mathbf{n}=\mathbf{6 4})\end{array}$ & $\mathbf{p}$ value \\
\hline CT progress in the first week & $98(76.56)$ & $39(60.94)$ & $59(92.19)$ & 0.000 \\
\hline CT absorption time & $21(16-29)$ & $19(15-23)$ & $26(16-34)$ & 0.001 \\
\hline
\end{tabular}

CT — computed tomography; N/A — not available

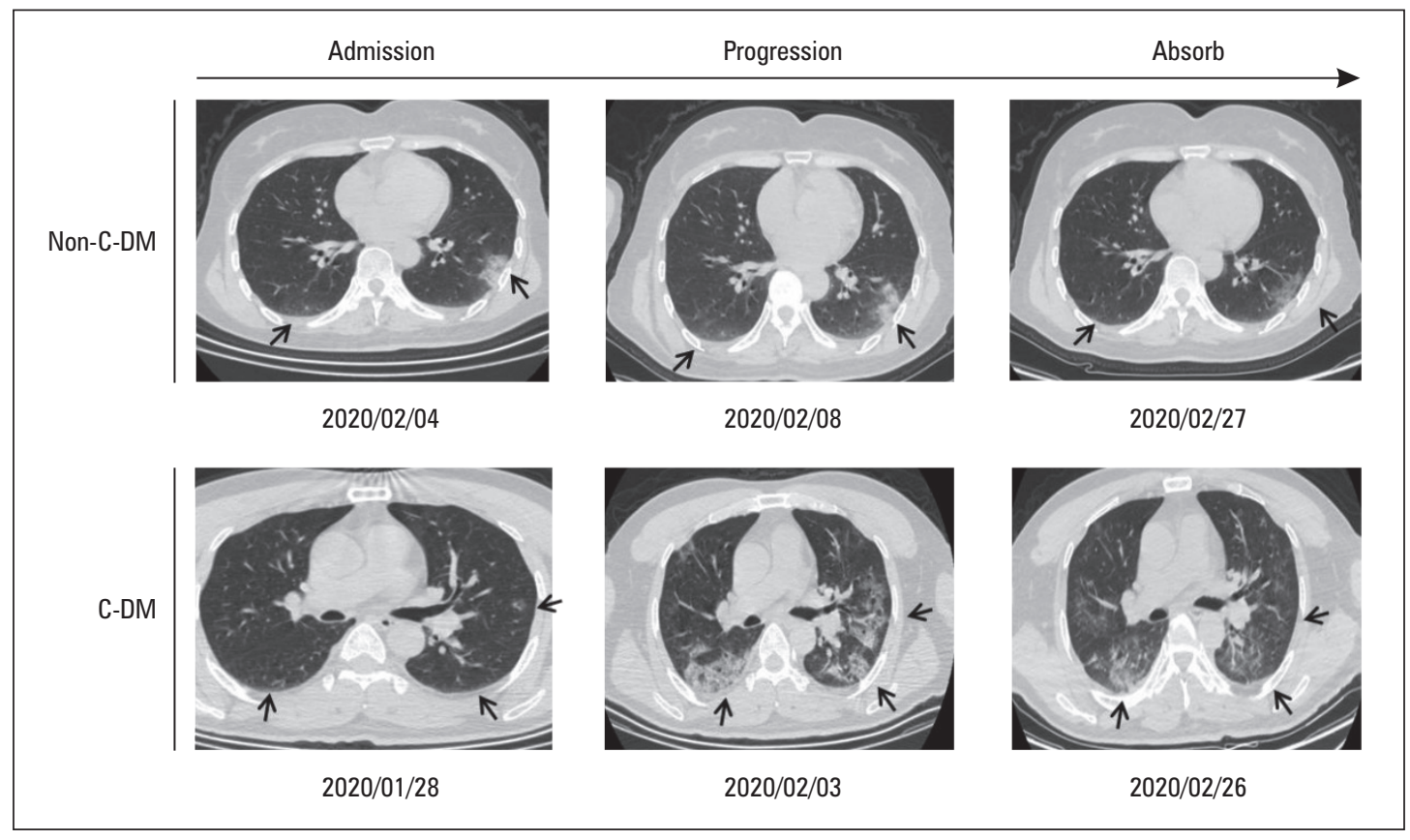

Figure 1. CT imaging of progression and absorption of pulmonary lesions in patients with diabetes (C-DM) and without diabetes (Non-C-DM)

\section{Discussion}

Coronaviruses are mostly round or oval in shape. They are named as coronaviruses because of their crown like appearance under an electron microscope. They can be divided into four genera: $\alpha, \beta, \gamma$, and $\delta$. COVID-19 belongs to the $\beta$ genus, with a diameter of $60-140 \mathrm{~nm}$. Coronaviruses are mainly caused by binding with receptors in patients [13]. Lu et al. found that the receptor binding domain (RBD) of COVID-19 was similar to that of SARS CoV, through the construction of a homologous structure model. Hoffmann et al. proved that the receptor of SARS CoV - angiotensin converting enzyme 2 (ACE2) was also the cellular receptor of COVID-19, and it needed the participation of cytoprotease TMPRSS2 to complete the invasion [14, 15]. The COVID-19 mainly destroyed alveoli and deep bronchial epithelial cells, and its pathological features were mainly inflammatory infiltration. These pathological features are consistent with our statistical laboratory examination results, clinical features, and CT imaging features.
According to the general situation of patients, we could observe that patients with COVID-19 complicated with diabetes mellitus were more likely to have unstable vital signs, decreased oxygen saturation, rapid heart rate, etc. The laboratory test results showed that patients with COVID-19 complicated with diabetes mellitus were more likely to have inflammatory reactions and hypercoagulable blood, and most of them were in severe condition at the time of admission. Previous studies also suggested that after COVID-19 virus invasion of patients, the results of laboratory examination were mostly showed the decrease of peripheral lymphocytes and the passive activation of the immune system [16]. This was consistent with the statistical results in this present study. SARS-related studies have shown that blood glucose fluctuations play a positive role in promoting the generation of inflammatory storms and the poor prognosis of clinical outcomes [17]. Patients with diabetes mellitus complicated with COVID-19 can be hyperglycaemic due to improper diet, irregular use of hypoglycaemic 
drugs, and glucocorticoid drugs and other factors that affect blood sugar. Hyperglycaemia can further reduce lymphocytes, thereby causing overactivation of the immune system, while excessive activation and improper activation of the epidemic free system can cause inflammatory storm, which is often the reason for severe clinical symptoms [18]. Some studies also suggest that blood glucose can be increased after COVID-19 virus invasion, and high blood glucose level is a high risk factor for disease progression. People with diabetes are more likely to be infected with $\mathrm{CO}$ VID-19 virus than people without diabetes because of their immune deficiency. When the virus invades, it mainly acts on the complement system. Complement immunity is one of the main mechanisms of humoral immunity. The complement system activates and mediates the production of antibodies at the time of the virus invading the body. However, in diabetic patients with immunodeficiency, the response to complement activation is decreased, and glycosylation can affect the expression of receptors in the complement activation system, resulting in diabetic patients being more susceptible to infection, and they are prone to change from mild patients to severe patients, with a higher mortality rate [19]. This was also consistent with the clinical outcome of this study that patients with $\mathrm{CO}$ VID-19 complicated with diabetes mellitus were more likely to develop respiratory failure and have a higher mortality rate than those without diabetes mellitus.

Compared with Non-C-DM, C-DM patients presented more lung segment and lobe infections on CT imaging. The area around the lesion was blurred and the boundaries were unclear. Lesions were found in the centre and around the lobes of both lungs, with a wide range, indicating severe lesions in C-DM patients. Studies found that COVID-19 mainly destroyed alveoli and deep bronchial epithelial cells, and its pathological features were mainly inflammatory infiltration. Under electron microscopy, tracheal epithelial cells were swollen, local cilia disappeared, and diffuse alveolar destruction was observed. COVID-19 caused exudation of high protein fluid, formation of a clear membrane, obvious monocyte infiltration, bronchiole were filled with cell debris, and there was apparent alveolar collapse with haemorrhage $[20,21]$. The pathological characteristics were similar to those caused by SARS and Mars coronavirus, but the degree of fibrosis and consolidation was weaker than that caused by SARS, which was consistent with the characteristics of our CT imaging statistics in this study. Most of the CT imaging of COVID-19 patients are cumulative double lung, with lesions distributed in and around the centre of both lungs, and the lesion range is more than $3 \mathrm{~cm}$.
According to the early autopsy results, the deep airway of the deceased patient was blocked by a large number of sputum plugs, which was consistent with the clinical manifestations [22]. Early studies confirmed that respiratory failure and circulatory failure were the main causes of death in COVID-19 patients [23]. The statistical results of this study showed that the proportion of C-DM patients complicated with respiratory failure during hospitalisation was higher than that of Non-C-DM and was statistically significant. In addition, it could also be found that C-DM patients had more severe CT imaging manifestations and a wide range of lesions, consistent with the clinical outcome.

\section{Conclusion}

To sum up, we reported 128 confirmed patients with COVID-19 infection and explored the effect of diabetes on COVID-19 patients based on clinical indicators and CT imaging features. We have provided a theoretical basis and clinical guidance for the treatment of patients with COVID-19 complicated with diabetes mellitus.

\section{Authors' contributions}

Y.Y and F.Y. contributed equally to the work.

\section{References}

3. Weiss SR, Leibowitz JL. Coronavirus pathogenesis. Adv Virus Res. 2011; 81: 85-164, doi: 10.1016/B978-0-12-385885-6.00009-2, indexed in Pubmed: 22094080.

4. Masters PS, Perlman D. Coronaviridae. In: Knipe CD, Howley PM. ed. Fields virology. 6th ed. Lippincott Williams \&Wilkins, Philadelphia 2013: 825-858.

5. Su S, Wong G, Shi W, et al. Epidemiology, Genetic Recombination, and Pathogenesis of Coronaviruses. Trends Microbiol. 2016; 24(6): 490-502, doi: 10.1016/j.tim.2016.03.003, indexed in Pubmed: 27012512.

6. Cui J, Li F, Shi ZL. Origin and evolution of pathogenic coronaviruses. Na Rev Microbiol. 2019; 17(3): 181-192, doi: 10.1038/s41579-018-0118-9, indexed in Pubmed: 30531947

7. Wong G, Liu W, Liu Y, et al. MERS, SARS, and Ebola: The Role of Super-Spreaders in Infectious Disease. Cell Host Microbe. 2015; 18(4): 398-401, doi: 10.1016/j.chom.2015.09.013, indexed in Pubmed: 26468744.

8. Guan WJ, Ni ZY, Hu Yu, et al. China Medical Treatment Expert Group for Covid-19. Clinical Characteristics of Coronavirus Disease 2019 in China. N Engl J Med. 2020; 382(18): 1708-1720, doi: 10.1056/NEJMoa2002032, indexed in Pubmed: 32109013.

9. Zhang C, Shi L, Wang FS. Liver injury in COVID-19: management and challenges. Lancet Gastroenterol Hepatol. 2020; 5(5): 428-430, doi: 10.1016/S2468-1253(20)30057-1, indexed in Pubmed: 32145190.

10. Singer M, Deutschman CS, Seymour CW, et al. Sepsis Definitions Task Force. Assessment of Clinical Criteria for Sepsis: For the Third International Consensus Definitions for Sepsis and Septic Shock (Sepsis-3). JAMA. 2016; 315(8): 762-774, doi: 10.1001/jama.2016.0288, indexed in Pubmed: 26903335.

11. Sharma S, Adrogue JV, Golfman L, et al. Intramyocardial lipid accumulation in the failing human heart resembles the lipotoxic rat heart. FASEB J. 2004; 18(14): 1692-1700, doi: 10.1096/fj.04-2263com, indexed in Pubmed: 15522914.

12. Tang N, Li D, Wang X, et al. Abnormal coagulation parameters are associated with poor prognosis in patients with novel coronavirus pneumonia. J Thromb Haemost. 2020; 18(4): 844-847, doi: 10.1111/jth.14768, indexed in Pubmed: 32073213.

13. Diagnosis and Treatment Protocol for Novel Coronavirus Pneumonia (Trial Version 7). Chin Med J. 2020; 133(9): 1087-1095, doi: 10.1097/cm9.0000000000000819, indexed in Pubmed: 32358325. 
14. Diabetes Branch of Chinese Medical Association, National Basic Diabetes Prevention and Management Office. [National basic diabetes prevention and management guidelines (2018)]. Chin J Int Med. 2018, 57(12): 885-893.

15. Kumar S, Maurya VK, Prasad AK, et al. Structural, glycosylation and antigenic variation between 2019 novel coronavirus (2019-nCoV) and SARS coronavirus (SARS-CoV). Virusdisease. 2020; 31(1): 13-21, doi: 10.1007/s13337-020-00571-5, indexed in Pubmed: 32206694.

16. Lu J, Gu J, Li K, et al. COVID-19 Outbreak Associated with Air Conditioning in Restaurant, Guangzhou, China, 2020. Emerg Infect Dis. 2020; 26(7): 1628-1631, doi: 10.3201/eid2607.200764, indexed in Pubmed: 32240078.

17. Fahmi M, Kubota Y, Ito M. Nonstructural proteins NS7b and NS8 are likely to be phylogenetically associated with evolution of 2019-nCoV Infect Genet Evol. 2020; 81: 104272, doi: 10.1016/j.meegid.2020.104272, indexed in Pubmed: 32142938.

18. Liu $Y$, Yang $Y$, Zhang $C$, et al. Clinical and biochemical indexes from 2019-nCoV infected patients linked to viral loads and lung injury. Sci China Life Sci. 2020; 63(3): 364-374, doi: 10.1007/s11427-020-1643-8, indexed in Pubmed: 32048163.

19. Yang JK, Feng Y, Yuan MY, et al. Plasma glucose levels and diabetes are independent predictors for mortality and morbidity in patients with
SARS. Diabet Med. 2006; 23(6): 623-628, doi: 10.1111/j.1464-5491.2006. 01861.x, indexed in Pubmed: 16759303

20. Ma RCW, Holt RIG. COVID-19 and diabetes. Diabet Med. 2020; 37(5): 723-725, doi: 10.1111/dme.14300, indexed in Pubmed: 32242990.

21. Klonoff DC, Umpierrez GE. Letter to the Editor: COVID-19 in patients with diabetes: Risk factors that increase morbidity. Metabolism. 2020; 108: 154224, doi: 10.1016/j.metabol.2020.154224, indexed in Pubmed: 32275971.

22. Chan JF, Zhang A, Yuan S, et al. Simulation of the clinical and pathological manifestations of Coronavirus Disease 2019 (COVID-19) in golden Syrian hamster model: implications for disease pathogenesis and transmissibility. Clin Infect Dis. 2020; [ahead of print], doi: 10.1093/cid/ciaa325, indexed in Pubmed: 32215622

23. Liu Q, Wang RS, Qu GQ, et al. [Gross examination report of a COVID-19 death autopsy]. Fa Yi Xue Za Zhi. 2020; 36(1): 21-23.

24. Ding YQ, Bian XW. [Analysis of coronavirus disease-19 (COVID-19) based on SARS autopsy]. Zhonghua Bing Li Xue Za Zhi. 2020; 49(4) 291-293.

25. Hanley B, Lucas SB, Youd E, et al. Autopsy in suspected COVID-19 cases. J Clin Pathol. 2020; 73(5): 239-242, doi: 10.1136/jclinpath-2020-206522, indexed in Pubmed: 32198191. 